\title{
Research Challenges and Opportunities in 5G Network
}

\author{
Shailendra Mishra ${ }^{1}$ and Mayank Singh ${ }^{2}$ \\ ${ }^{1}$ College of Computer and Information Sciences, Majmaah University, KSA \\ ${ }^{2}$ Krishna Engineering College, India
}

\begin{abstract}
The term $5 G$ is sometimes used to encapsulate all existing technologies for all time availability, higher coverage area and higher network density with respect to cell and devices.5G system will be compatible with existing technologies such as $2 G, 3 G, 4 G$, WiFi etc. and will support more than ten thousand times data traffic compare to $4 G$ and data downloading more than 1000 times compare to 4G. It will run on Ultra High Spectrum Band that will carry the future Internet, it is not only evolution but it is a revolution in communication era. In this paper we present several emerging technologies that support $5 G$ standard along with research problems and challenges for $5 G$ systems.
\end{abstract}

Keywords: $M I M O, 5 G, W N V, C-R A N, D 2 D, M 2 M$

\section{Introduction}

Third generation cellular systems are basically designed for multimedia communication while $4 \mathrm{G}$ technology provides ultra-broadband access for mobile devices. 4G technology is based on internet protocol (IP) \& OFMDA multi-carrier transmission methods and uses packet switching technology. Peak data rates of $100 \mathrm{Mb} / \mathrm{s}$ are supported in the downlink as well as in uplink in $20 \mathrm{MHz}$ channel in 4G. IEEE 802.16 (Wi-Max) is a representative standard of $4 \mathrm{G}$ as it supports all-IP services (including voice, messaging) besides supporting both TDD and FDD operations. 3G and 4G wireless technologies were driven by demand for data services over the Internet.

5G network consists of computing nodes and small cell like pico cells for effective communication between devices. This network will be capable of load balancing, minimizing the interference, power adaptation etc. Nodes (Base stations, user equipment's) are heterogeneous in nature and will be integrated through a cloud-based network [1]. Communication scheme in $5 \mathrm{G}$ network will support full duplex mode (FD). By using FD communication scheme, spectral efficiency will be doubled at the physical layer $[2,3]$. Radio Frequency (RF) signals carry energy and information simultaneously. Also 5G network will optimize the energy [4]. Cloud-based radio access network (CRAN) will be used to reduce the capital expenditure and operational expenditure. C-RAN will make scheduling relatively easy [5]. Sharing the common resources like network infrastructure and licensed spectrum, RAN is possible by using wireless network virtualization (WNV) to reduce capital as well as operational expenditure [6].

Three criteria for the 5G standard have been established which are (a) 1Gbps end to end data rate to start with and multi-gigabits in future (b) latency (end-to-end round trip delay) of one millisecond and (c) more energy efficiency than its predecessors [7]. Major technology requirements for $5 \mathrm{G}$ wireless cellular networks are reported in [1, 8, 9 and 10] for compatibility of existing cellular network as well as for virtualization of wireless resources.

Major 5G technology components are MULTI-ANTENNA TRANSMISSION (MIMO), ULTRA RADIO ACCESS DESIGN (RAN), and FLEXIBLE DUPLEX FDD reported in [11] 
MULTI-ANTENNA TRANSMISSION (MIMO): MIMO technology already plays an important role for $4 \mathrm{G}$ wireless communication system and will play important role in the 5G communication system, i.e., for operation at higher frequencies as well as the use of MIMO System for beam forming to support the system at high as well as low frequency.

ULTRA RADIO ACCESS DESIGN (RAN): It will be used to transfer high data rate from device to device, it will also include synchronization mechanism for signals, network acquisition and estimation techniques.

FLEXIBLE DUPLEX FDD (Frequency Division Duplex) concept will also incorporate, to share the medium with minimum or least interference. For lower frequency Band FDD will be used and for higher i.e., more than $10 \mathrm{GHz}$ TDD(Time Division Multiplexing ) will be used, for dense deployment of cell as well as devices.

MIMO technology will arrive in a big way with 5G System.

In multiple input-multiple output (MIMO) system, "multiple in" means a WLAN device simultaneously sends two or more radio signals into multiple transmitting antennas. "Multiple out" refers to two or more radio signals coming from multiple receiving antennas. These views of "in" and "out" may seem reversed, but MIMO terminology focuses on the system interface with antennas rather than the air interface. MIMO technology offers tremendous performance gains for wireless LANs at relatively low cost. Any system with multiple inputs into the receiver and multiple outputs to the transmitter is a MIMO system, but implementing such a system involves several distinctly different radio techniques. Some of these techniques are beneficial and fully compatible with today's standard WLAN equipment, while others do not improve performance when used with existing equipment. MIMO transceiver techniques promise tremendous performance benefits for wireless systems in terms of capacity increase and diversity gain, and MIMO extensions have been proposed to virtually every contemporary wireless communication system $[12,13]$.

MIMO transceiver techniques promise tremendous performance benefits for wireless systems in terms of capacity increase and diversity gain, and MIMO extensions have been proposed to virtually every contemporary wireless communication system [14].

In MIMO, "multiple in" means a WLAN device simultaneously sends/transmit and receive more radio signals using multiple transmitters. In MIMO there are multiple transmit antennas as well as receiver antennas, single information stream is split in to multiple streams and transmitted over multiple antennas in the same frequency band, resulting increase in data throughput $\&$ link range, reduce bit error rates, and minimize the power, on receiving end having multiple receivers result in increase in the amount of received power and it also reduces the problem of multipath using principle of maximal combining.

A scattering channel can be problematic because it causes interference and hence suboptimal reception. Many methods have been introduced to counter the effect of interference, such as interference cancellation, phased array antennas and many of the diversity techniques However, it is possible to exploit the spatial diversity aspects of the channel, especially when it is highly dispersive and causes a reasonable degree of scattering. The MIMO concept is designed to take advantage of the channel dispersive nature instead of counteracting it. There are various techniques that work with a MIMO system. Spatial multiplexing for example works by splitting the data stream to multiple streams (equal to number of transmitting antennas) [15].

If the channels are totally independent and uncorrelated it is possible to increase the overall data rate by multiple folds. Another method is to employ space-time coding where the transmitted data is sent over multiple antennas at different times. With a MIMO 
system, the combined effect is a reduced power requirement (signal to noise ratio), or an increased throughput if traded off with power.

Pilot contamination problem can be eliminated using Very large MIMO System. Very large MIMO saving transmit power As the number of base station antennas grows, the system gets almost entirely limited from the reuse of pilots in neighboring cells, the so called pilot contamination concept [16].

Massive-MIMO systems can be termed as the scenario of multi-user MIMO in which the number of transmitter terminals is very less than the number of BS (base station) antennas. Massive MIMO networks can provide higher performance than partial multiuser MIMO since the multiple antennas used are much smarter, massive MIMO techniques increase in data bandwidth without any extra transmission power [17].

Massive MIMO technology is used for energy and spectral efficiency that includes small-scale fading and improve the spectral and energy efficiency with orders of magnitude compared to a single-antenna system [18]. In massive MIMO spatial division and multiplexing (JSDM) allow for a large number of antennas at the base station while requiring reduced-dimensional channel state information at the transmitter (CSIT).

\section{Literature Review}

In order to meet the objective, the review of literature will be carried out in the following contexts

-Multi-antenna arrays scheme.

- System models to calculate channel estimation, pilot contamination, path loss \& antenna correlation and capacity estimation

-Energy efficient protocol and resource allocation scheme

The First Generation (1G) Cellular System was developed to offer wireless voice services based on analog technology, Frequency modulation, Frequency Division Multiple Access FDMA) and digital signaling techniques are used, each channel was assigned to a unique frequency band within a cluster of cells. Representative systems of $1 \mathrm{G}$ are NAMPS, TACS and Nordic Mobile Telephone System (NMT-900). All the second-generation systems are based on digital modulation techniques. In 2nd Generation system Time Division Multiple Access (TDMA) and Code Division Multiple Access (CDMA) are used along with FDMA.

Third generation cellular systems are being designed for multimedia communication, with them person-to-person communication can be enhanced with high quality images and video. In the standardization forums, WCDMA technology has emerged as the most widely adopted third air interface. Its specification has been created in 3GPP (The ThirdGeneration Partnership Project), which is the joint standardization project of the standardization bodies from Europe, Japan, Korea, the USA and China. Within 3GPP, WCDMA is called UTRA (Universal Terrestrial Radio Access). Frequency Division Duplex (FDD) and Time Division Duplex (TDD) are the name WCDMA being used to cover both FDD and TDD operation [27].

The information inside the WCDMA system is spread over a band of approximately 5 $\mathrm{MHz}$ and is thus give a name wideband CDMA or WCDMA. Access to information and services on public and private networks will be enhanced by the higher data rates and new flexible communication capabilities of third generation systems.

The primary requirements of the third generation cellular systems are voice quality, high data rate, support of both packet and circuit-switched data services, usage of the available radio spectrum, backward compatibility with pre-existing networks and flexible to new services and technology. The concept of multiple antennas was first introduced in 
1960 and applied in military radar system for finding the direction and separating one signal to other.

Third generation cellular systems are designed for multimedia communication, while 4G technology provides ultra-broadband access for mobile devices, based on internet protocol (IP) \& OFMDA multi-carrier transmission methods and uses packet switching technology, peak data rates of $100 \mathrm{Mb} / \mathrm{s}$ are supported in the downlink as well as in uplink in $20 \mathrm{MHz}$ channel in 4G. IEEE 802.16 (Wi-Max) is representative standards of 4G, it supports all-IP services (including voice, messaging) and supported both TDD and FDD operation [19].

The Wireless LAN standard IEEE 802.11a can at most offer 54Mbps via occupying $16.6 \mathrm{MHz}$ bandwidth; that is to say, utilized spectral efficiency is about $3.25 \mathrm{bits} / \mathrm{Hz} / \mathrm{sec}$ for the best transmission case. The present experiment of MIMO system such as IEEE 802.11n (Wi-Fi) uses MIMO-OFDM which ensures the usage of multiple receive antennas often make use of four transmit antennas [20,25].

IEEE $802.16 \mathrm{~m}$ supports very low end-to-end round trip delay i.e., latency of approx.10-30 ms, data rate of $1 \mathrm{Mbps}$ up to $250 \mathrm{~km} / \mathrm{h}$ [21]. Network Function Virtualization (NFV) is a network architecture that separates the hardware from software or 'function'. Software Defined Networks (SDN) is an extension of NFV wherein software can perform dynamic reconfiguration of an operator's network topology to adjust to load and demand. Most of LTE networks are using NFV and SDN as the Heterogeneous Networks (HetNets) [22].

John Thompson et al. discussed upcoming 5G unified wireless technology that will be fully implemented in 2020. 5G systems are likely to be much more diverse and used to encapsulate all existing technologies for all time availability, higher coverage area and higher network density with respect to cell and devices, and will support more than ten thousand times data traffic compared to $4 \mathrm{G}$ and data downloading is more than 1000 times compare to 4G. It will run on Ultra High Spectrum Band i.e., $70 \mathrm{GHZ}$ or more. [23].

G. J. Foschini et al., proposed a multi-antenna arrays scheme. By using multi-antenna arrays scheme transmission and reception can lead to significantly increased bit-rates [24]. Jian Xu et al., provided a comprehensive resource management algorithm [26]. The 3rd generation partnership project's (3GPP) LTE, discusses migration of $3 \mathrm{G}$ to $4 \mathrm{G}$. 4G systems are based on OFDM scheme, using MIMO technology and supported both TDD and FDD operation. Peak data rates of $100 \mathrm{Mb} / \mathrm{s}$ are supported in the downlink as well as in uplink in $20 \mathrm{MHz}$ channel [27].

J. Hoydis et al., proposed a system model for Massive MIMO system to calculate channel estimation, pilot contamination, path loss and antenna correlation, also proposed an architecture and precoding scheme that will be deployed using macro base stations (BSs) with very large antenna arrays [28]. F. Rusek et al., discussed and eliminated the pilot contamination problem using very large MIMO system. Simulations results illustrate that very large MIMO systems are saving transmit power. [29].

H. Q. Ngo et al., discussed massive MIMO in terms of energy and spectral efficiency and quantified them for a channel model that includes small-scale fading and illustrated that large antenna arrays can improve the spectral efficiency as well as energy efficiency [30].H. Yin et al. proposed an approach to deal with the pilot contamination problem and also discussed the channel estimation scheme in multi-cell interference-limited cellular networks [31].

Shailendra Mishra et al., proposed an resource management algorithm for MIMO System. The objective of the resource management is formulated into a constrained optimization problem. The resource management algorithm optimizes subcarrier allocation, power distribution, and bit distribution for all users jointly according to the instantaneous channel state information (CSI) and QoS requirements. The presence of multiple antennas generates another DOF in the space domain. At the 
same time, it introduces co channel interference (CCI) since different users are allowed to transmit using the same sub-carriers and time slots [32].

K. T. Truong et al., discussed the effects of Channel Aging in Massive MIMO Systems and proposed channel prediction scheme to overcome the negative effects of channel aging [33]. A. Adhikary et al., proposed joint spatial division multiplexing scheme (JSDM) [34].

Regarding 5G challenges, Federico Boccardi et al., said that Network design should be device-centric rather than cell-centric and should be focused on the needs of user equipment, identified five major directions for future research and standardization i.e., Network design should be device-centric rather than cell-centric and should be focused on the needs of user equipments.

$5 \mathrm{G}$ is based on higher-frequency millimeter wave bands where much more bandwidth is available. Massive MIMO as a key 5G technology needs intelligent terminals that are capable of mitigating interference and communicating directly with other devices without network assistance and it supports machine-to-machine communications in an effective and integrated manner [35].

Boyd Bangerter et.al., discussed the paradigm shifts regarding change in cellular network layout, due to the growth of heterogeneous network architectures, need of new technologies such as massive MIMO, full duplex technology, device-to-device communications and user terminal design and requirements [36]. Cheng Xiang Wang et al. discussed and identified key technologies for $5 \mathrm{G}$ systems for promoting greener communication [37].

E. G. Larsson et al., discussed the massive MIMO technology concept. Massive MIMO networks can provide higher performance than partial multi-user MIMO since the multiple antennas used are much smarter. Massive MIMO techniques result in increase in data bandwidth without any extra transmission power. This paper also discussed the factors that limit massive MIMO such as pilot contamination, imperfect channel reciprocity etc [38].

Y. Kim et al., discussed the application of massive MIMO in $4 \mathrm{G}$ cellular system in terms of deployment scenarios and antenna array implementation [40]. J Choi et al. proposed an open-loop training frameworks for channel estimation and closed-loop training frameworks for channel prediction for FDD-based massive MIMO System [39].

Al Muthanna et al., proposed a method for capacity estimation for 5G cellular networks using field-level simulations. Results depicted in this paper show that by reducing antenna beam width from $65^{\circ}$ to $30^{\circ}$, capacity can enhance roughly by 3.0 times at a distance of $220 \mathrm{~m}$ from the base station (BS) [40].

Gang Liu et al., discussed both in-band full duplex relay (FDR) and wireless virtualization promising technologies for $5 \mathrm{G}$ cellular networks and proposed a virtual resource management architecture for in-band FDR networks. Simulation results illustrated the effectiveness of the proposed scheme [41].

$\mathrm{H}$. Ju et al., proposed and designed an energy efficient protocol and resource allocation scheme, to support simultaneous wireless energy transfer in the uplink as well as downlink. In the proposed optimal resource allocation scheme one hybrid access point (H-AP) operate in full duplex (FD) and broadcasts wireless energy to a set of distributed users in the downlink (DL) and, at the same time, receives independent information from the users via time-division multiple access in the uplink (UL) [42].

Ping Wang et. al., discussed the aim and design of 5G cellular networks to achieve ultra-low latency for real-time video streaming service through heterogeneous environments. Also proposed cell association and antenna allocation algorithms that ensure the users and the cells cannot gain higher data [43]. 
Almuthanna et al., proposed a method for capacity estimation for $5 \mathrm{G}$ cellular networks using field-level simulations. Results depicted in this paper shows that by reducing antenna beamwidth from $65^{\circ}$ to $30^{\circ}$, capacity can enhance roughly by 3.0 times at a distance of $220 \mathrm{~m}$ from the base station (BS) [44]

To conclude, in spite of many issues and challenges regarding successful implementation of $5 \mathrm{G}$, visual communication will become prominent, as if we will be meeting face to face and it will benefit the mankind, since it can minimize problems in many ways not just in terms of speed and security but also in terms of increased number of connected devices and high efficiency. Also, very less or no evidence is published that can show the drawbacks of $5 \mathrm{G}$.

\section{Research Issues}

- 5G wireless communication systems will consist of base stations(BSs) having more than 100 antennas to enable massive MIMO for creating beams to multiple users and UEs will be operating in mm-wave frequency bands for enabling heavy data traffic. If the UE also uses beam-forming antenna arrays using digital signal processing, battery life of UEs can be reduced.

- 5G network requires high data rates and capacity; it requires air interface technology based on new other modulation schemes. Which modulation scheme can be used to achieve more than 1 Gbps data rate?

- 5G systems will use RF and mixed signal major issues are RFIC integration and millimeter wave packaging. Radio Frequency Integrated Circuit (RFIC) integration, In RFIC integration, integration of the front-end module with mixed signal (digital, analog or hybrid beamforming) require highly integrated solutions, while in millimeter wave packaging, question is how to place multiple antennas and reduce electrical as well as thermal effect.

- How to optimize energy efficiency and throughput in end-to-end communication?

- How to allocate the total transmission energy into parallel coded-channels of the MIMO system so that upper-bound throughput can be achieved?

- How to decrease end-to-end delay for better performance and functionality?

- Channel model evaluation for 5G technologies is to be done.

- How to deal with the correlated channel in the massive MIMO system?

- How to measure the real correlation by some existent or newer algorithmic implementations?

- How to design Resource Allocation \& scheduling Algorithms for $5 \mathrm{G}$ Network to optimize throughput and enhance the capacity of cellular cell?

\section{Conclusion}

This paper deals with the challenges and opportunities in 5G System. The main target of researchers is how to increase the user peak data rates and quality of service (QoS), and in general to increase the Spectral Efficiency, Energy efficiency, Capacity enhancement along with Hardware efficiency in Ad-hoc as well as microcellular environment.

$4 \mathrm{G}$ technologies provide ultra-broadband access for mobile devices. $4 \mathrm{G}$ networks are based on Internet protocol (IP) and uses packet switching technology \& OFMDA multi-carrier transmission methods, Long term Evolution, IEEE 802.16 (WiMax) are representative standards of $4 \mathrm{G}$. It supports all-IP services (including voice, messaging). The 3rd generation partnership project's (3GPP) LTE discusses 
migration of $3 \mathrm{G}$ to $4 \mathrm{G}$. It is based on OFDM scheme and using MIMO technology and supported both TDD and FDD operation. Peak data rates of $100 \mathrm{Mb} / \mathrm{s}$ are supported in the downlink as well as in uplink in $20 \mathrm{MHz}$ channel.

The Wireless LAN standard IEEE $802.11 \mathrm{a}$ can at most offer 54Mbps via occupying $16.6 \mathrm{MHz}$ bandwidth; that is to say, utilized spectral efficiency is about $3.25 \mathrm{bits} / \mathrm{Hz} / \mathrm{sec}$ for the best transmission case. The present experiment of MIMO system such as IEEE 802.11n (Wi-Fi) uses MIMO-OFDM which ensures the usage of multiple receive antennas often make use of four transmit antennas, is capable of achieving throughput up to $300 \mathrm{Mb} / \mathrm{s}$ utilizing two spatial streams in a $40 \mathrm{MHz}$ channel in the $5 \mathrm{GHz}$ Band. IEEE $802.16 \mathrm{~m}$ enable interoperability between WiMAX and 3GPP's Long Term Evolution (LTE) support high-speed mobile wireless access (up to $350 \mathrm{~km} / \mathrm{h}$ ) and peak data rates of over $300 \mathrm{Mb} / \mathrm{s}$ using 4x 4 MIMO.

The IEEE 802.20 Mobile Broadband Wireless Access (MBWA) standard supports very low end-to-end round trip delay i.e., latency (approx.10-30 ms), data rate 1 Mbps upto $250 \mathrm{~km} / \mathrm{h}$. Owing to the advancement of RF component manufacturing, it is possible that the future high data-rate-demand communication system would require more transmit antennas to overcome the bottleneck of limited capacity. A potential solution is to assess the possible capacity improvement. If it is much larger than the real needs, then increase the power (to raise the SNR) instead of increasing a transmit antenna. Such a problem involving joint resource allocation is also interesting.

Fifth generation (5G) systems are likely to be much more diverse and used to encapsulate all existing technologies for all time availability, higher coverage area and higher network density with respect to cell and devices, and will support more than ten thousand times data traffic compare to $4 \mathrm{G}$ and data downloading is more than 1000 times compare to $4 \mathrm{G}$.However, the implementation of $5 \mathrm{G}$ system needs to measure; How to optimize energy efficiency and throughput in end-to-end communication in the infrastructure networks, How to allocate the total transmission energy into parallel codedchannels of the multiple input multiple output (MIMO) system, so that upper-bound throughput can be achieved and how to deal with the correlated channel in the MIMO system and also needs to measure the real co-relation by some existent or newer algorithmic implementations.

\section{References}

[1] S. Ruepp Zakrzewska and M. Berger, "Towards converged 5G mobile networks - Challenges and current trends", Proceedings of the ITU Kaleidoscope Academic Conference, (2014) June, pp. 39-45.

[2] J. Choi, M. Jain, K. Srinivasan, P. Levis and S. Katti, "Achieving single channel, full duplex wireless communication", Proceedings of the International Conference on Mobile Computing and Networking, (2010), pp. 1-12.

[3] S. Hong, J. Brand, J. Choi, M. Jain, J. Mehlman, S. Katti and P. Levis, “Applications of self-interference cancellation in 5G and beyond", IEEE Communications Magazine, vol. 52, no. 2, (2014) February, pp. 114-121.

[4] L. Xiao, P. Wang, D. Niyato, D. Kim and Z. Han, "Wireless networks with RF energy harvesting: A contemporary survey", IEEE Communications Surveys \& Tutorials, (2014).

[5] H.-L. Checko, Y. Yan Christiansen, L. Scolari, G. Kardaras, M.-S. Berger and L. Dittmann, "Cloud RAN for mobile networks - A technology overview", IEEE Communications Surveys \& Tutorials, (2014).

[6] Liang and F.-R. Yu, "Wireless network virtualization: A survey, some research issues and challenges", IEEE Communications Surveys \& Communications Surveys \& Tutorials, IEEE, vol. 17, no. 1, (2015).

[7] http://www.techrepublic.com/article/does-the-world-really-need-5g

[8] E. Hossain, "5G Cellular: Key Enabling Technologies and Research Challenges", IEEE Instrumentation and Measurement Magazine, vol. 18, no. 3, (2015).

[9] P. Demestichas, A. Georgakopoulos, D. Karvounas, K. Tsagkaris, V. Stavroulaki, J. Lu, C. Xiong and J. Yao, "5G on the horizon: Key challenges for the radio-access network", IEEE Vehicular Technology Magazine, vol. 8, no. 3, (2013) September, pp. 47-53.

[10] http://www.ericsson.com/res/docs/whitepapers/wp-5g.pdf 
[11] D. Hossain Niyato, E. Wavegedara and K. CBhargava, "Radio Link Level Performance in Multi-Rate MIMO Wireless Networks: Analysis and Optimization", Wireless Comm. and Networking Conference, 2007.WCNC 2007, IEEE, (2007) March, pp. 294-298.

[12] S. Mishra and D. S. Chauhan, "Link Level Performance of Multiple input Multiple Output system", International Journal of Control and Automation, vol. 2, no. 3, (2009) September, pp. 29-41.

[13] J. Kim and J. M. Cioffi, "Spatial multiuser access with antenna diversity using singular value decomposition", Proceedings IEEE Int. Conf. Communication., vol. 3, (2000) June, pp. 1253-1257.

[14] S. M. Alamouti, "A Simple Transmitter Diversity Scheme for Wireless Communications", IEEE J. Sel. Area Comm., (1998) October, pp. 1451-1458.

[15] K. T. Truong and R. W. Heath, "Effects of Channel Aging in Massive MIMO Systems", Journal of Communications and Networks (JCN), vol. 15, no. 4, (2013) August, pp. 338-351.

[16] F. Boccardi, R. W. Heath Jr., A. Lozano, T. L. Marzetta and P. Popovski, "Five Disruptive Technology Directions for 5G”, IEEE Communications Magazine, vol. 52, no. 2, (2014), February, pp. 74-80.

[17] J. Choi, Z. Chance, D. J. Love and U. Madhow, "Noncoherent Trellis Coded Quantization: A Practical Limited Feedback Technique for Massive MIMO Systems", IEEE Transactions on Communications, vol. 61, no. 12, (2013) December, pp. 5016-5029.

[18] V. Jungnickel, K. Manolakis, W. Zirwas, B. Panzner, V. Braun, M. Lossow, M. Sternad, R. Apelfröjd, and T. Svensson, "The Role of Small Cells, Coordinated Multipoint, and Massive MIMO in 5G", IEEE Communications Magazine, vol. 52, no. 5, (2014) May, pp. 44-51.

[19] 3GPP TS 36.201 V8.1.0 (2007-11), "LTE physical layer - general description (Release 8)", 3GPP TSG RAN, (2007).

[20] IEEE Std 802. 16e $\mathrm{e}^{\mathrm{TM}}-2005$ \& IEEE Std 802. 16e $\mathrm{e}^{\mathrm{TM}}$ - 2004/Cor 1-2005, "IEEE standard for local and metropolitan area networks Part 16: Air Interface for fixed and mobile broadband wireless access systems amendment 2: Physical and medium access control layers for combined fixed and mobile operation in licensed bands and Corrigendum 1", (2006) February 28.

[21] IEEE Std 802.20 interface for mo-bile broadband wireless access systems supporting vehicular mobility-physical and media access control layer specification”, (2008) August 29.

[22] https://portal.etsi.org/nfv/nfv_white_paper.pdf.

[23] J. Thompson, "5G Wireless Communication Systems: Prospects and Challenges", IEEE Communications Magazine, (2014) February.

[24] G. J. Foschini and M. J. Gans, "On limits of wireless communications in a fading environment when using multiple antennas", Wireless Personal Communications, vol. 6, (1998), pp. 311-335.

[25] Atheros Communications, Inc., "Getting the Most out of MIMO: Boosting Wireless LAN Performance with Full Compatibility", (2005), pp. 3-9.

[26] J. Xu, J. Kim, W. Paik and J. SooSeo, "Adaptive Resource Allocation Algorithm with Fairness for MIMO-OFDMA System”, 0-7803-9392-9/06(c), IEEE, (2006).

[27] 3GPP TS 36.201 V8.1.0 (2007-11), "LTE physical layer - general description (Release 8)", 3GPP TSG RAN, (2007)

[28] J. Hoydis, K. Hosseini, S. ten Brink and M. Debbah, "Making Smart Use of Excess Antennas: Massive MIMO, Small Cells, and TDD”, Bell Labs Technical Journal, vol. 18, no. 2, (2013) September, pp. 5-21.

[29] F. Rusek, D. Persson, B. K. Lau, E. G. Larsson, T. L. Marzetta, O. Edfors and F. Tufvesson, "Scaling up MIMO: Opportunities and Challenges with Very Large Arrays", IEEE Signal Processing Magazine, vol. 30, no. 1, (2013) January, pp. 40-46.

[30] H. Q. Ngo, E. G. Larsson and T. L. Marzetta, "Energy and Spectral Efficiency of Very Large Multiuser MIMO Systems", IEEE Transactions on Communications, vol. 61, no. 4, (2013) April, pp. 1436-1449.

[31] H. Yin, D. Gesbert, M. Filippou and Y. Liu, "A Coordinated Approach to Channel Estimation in LargeScale Multiple-Antenna Systems", IEEE Journal on Selected Areas in Communications, vol. 31, no. 2, (2013) February, pp. 264-273.

[32] S. Mishra and D. S. Chauhan, "Resource allocation for multi access MIMO System", International Journal of Mobile Computing and Multimedia Communications (IJMCMC), (2011) February.

[33] K. T. Truong and R. W. Heath, "Effects of Channel Aging in Massive MIMO Systems", Journal of Communications and Networks (JCN), vol. 15, no. 4, (2013) August, pp. 338-351.

[34] J. Nam Adhikary, J.-Y. Ahn and G. Caire, "Joint Spatial Division and Multiplexing The Large-Scale Array Regime", IEEE Transactions on Information Theory, vol. 59, no. 10, (2013) October, pp. 64416463.

[35] F. Boccardi, R. W. Heath Jr, T. L. Marzetta and P. Popovski, "Five Disruptive Technology Directions for 5G”, IEEE Communications Magazine, (2014) February.

[36] B. Bangerter, S. Talwar, R. Arefi and K. Stewart, "Networks and Devices for the 5G Era", IEEE Communications Magazine, (2014) February.

[37] C.-X. Wang, F. Haider, X. Gao and X.-H. You, "Cellular Architecture and Key Technologies for 5G Wireless Communication Networks", IEEE Communications Magazine, (2014) February.

[38] E. G. Larsson, F. Tufvesson, O. Edfors and T. L. Marzetta, "Massive MIMO for Next Generation Wireless Systems", IEEE Communications Magazine, vol. 52, no. 2, (2014) February, pp. 186-195. 
[39] Y. Kim, H. Ji, J. Lee, Y.-H. Nam, B. L. Ng, I. Tzanidis, Yi Li and J. Zhang, "Full Dimension MIMO (FD-MIMO): The Next Evolution of MIMO in LTE Systems", IEEE Wireless Communications, vol. 21, no. 2, (2014) April, pp. 26-33.

[40] A. MuthannaTurki Nassar, A. IyandaSulyman and A. Alsanie, "Radio Capacity Estimation for Millimeter Wave 5G Cellular Networks Using Narrow Beamwidth Antennas at the Base Stations", International Journal of Antennas and Propagation, (2015).

[41] G. Liu, F. R. Yu, H. Ji, V. C. M. Leung and X. Li, "In-band full-duplex relaying (FDR)for 5G cellular networks with wireless virtualization", Network, IEEE, vol. 29, no. 6, (2015) December.

[42] H. Ju and R. Zhang, "Optimal resource allocation in full-duplex wireless-powered communication network", IEEE Transactions on Communications, vol. 62, no. 10, (2014) October, pp. 3528-3540.

[43] W. Song, D. Niyato, P. Wang and Y. Xiao, "QoS-aware cell association in $5 \mathrm{G}$ heterogeneous networks with massive MIMO", Network, IEEE, vol. 29, no. 6, (2015) December.

[44] A. Turki Nassar, A. Iyanda Sulyman and A. Alsanie, "Radio Capacity Estimation for Millimeter Wave 5G Cellular Networks Using Narrow Beamwidth Antennas at the Base Stations", International Journal of Antennas and Propagation, Article ID 878614, 6 pages http://dx.doi.org/10.1155/2015/878614, vol. 2015, (2015). 
International Journal of Future Generation Communication and Networking Vol. 10, No. 6 (2017) 